\title{
Thermodynamic, Structural, Surface and Transport Properties of In-Tl Liquid Alloy at Different Temperatures
}

\author{
K. K. Mishra, H. K. Limbu, A. Dhungana, I. S. Jha, D. Adhikari* \\ Department of Physics, Mahendra Morang Adarsha Multiple Campus, Tribhuvan University, Biratnagar, Nepal \\ Email: *adksbdev@yahoo.com
}

How to cite this paper: Mishra, K.K., Limbu, H.K., Dhungana, A., Jha, I.S. and Adhikari, D. (2018) Thermodynamic, Structural, Surface and Transport Properties of In-Tl Liquid Alloy at Different Temperatures. World Journal of Condensed Matter Physics, 8, 91-108.

https://doi.org/10.4236/wjcmp.2018.83006

Received: June 25, 2018

Accepted: August 10, 2018

Published: August 13, 2018

Copyright $\odot 2018$ by authors and Scientific Research Publishing Inc. This work is licensed under the Creative Commons Attribution International License (CC BY 4.0).

http://creativecommons.org/licenses/by/4.0/

\begin{abstract}
The thermodynamic, structural, surface and transport properties of In-Tl binary liquid alloy are studied on the basis of theoretical analysis using the regular solution model at different temperatures. The properties of the alloy at $723 \mathrm{~K}$ have been computed by estimating the best fit value of order energy parameter $(\omega)$ in the entire range of concentration to match their observed and theoretical values. The values of order energy parameter at different temperatures have been calculated using the value of order energy parameter at $723 \mathrm{~K}$ which played key role to study different properties of the alloy using optimization method. The theoretical analysis gives the positive energy parameter $(\omega)$, which is found to be temperature dependent.
\end{abstract}

\section{Keywords}

In-Tl Alloy, Optimization, Segregation, Thermodynamic Properties, Structural Properties

\section{Introduction}

Various properties, such as hardness, corrosion resistance, wear resistance, mechanical strength, and fatigue strength, melting and boiling temperatures etc. of an alloy are different from its individual components. The properties of alloys in solid state are usually determined by thermodynamic, surface, structural, transport and electrical properties of alloys in liquid state. But there is complexity in determining the properties of liquid alloys due to lack of long range atomic order. Therefore several models [1]-[9] have long been proposed to understand the properties of alloys in liquid state.

The binary In- $\mathrm{Tl}$ alloy has widely been studied in the past because of its wide 
range of practical applications [10] [11] [12]. It is a shape memory alloy [13] having low melting temperature (fusible alloy). The indium based fusible alloy has the ability to wet and adhere to ceramic and glass surfaces. This characteristic can be useful in sealing and bonding of sensitive electronic devices, glass, ceramic and temperature-sensitive materials.

In this work we have used regular solution model [14] to explain thermodynamic and structural properties of In- $\mathrm{Tl}$ at $723 \mathrm{~K}$. In regular solution model, the interaction energy is considered as input parameter and is determined by fitting observed free energy of mixing at different concentrations. Thermodynamic behavior of the alloy is studied by computing free energy of mixing $\left(G_{M}\right)$, activity (a), entropy of mixing $\left(S_{M}\right)$ and heat of mixing $\left(H_{M}\right)$. Structural properties is interpreted by the concentration fluctuation in the long wavelength limit $\left(S_{c c}(0)\right)$ and chemical short range order parameter $\left(\alpha_{1}\right)$. The surface properties are studied by using Butler's model [15] and viscosity is studied with the help of Moelwyn-Hughes equation [16]. In order to compute all these properties at higher temperatures optimization procedure is used. Basic theoretical formulation of regular solution model has been presented in Section (2), result and discussion in Section (3) and conclusion are dealt in Section (4).

\section{Theoretical Formalism}

\subsection{Thermodynamic Properties}

Regular solution model [14] deals with the A-B alloy in liquid state in which the constituent atoms A and B are sufficiently similar in size and shape so that they are interchangeable on the lattice or quasi-lattice, and the configuration is no longer independent of the mutual disposition of the two or more kinds of molecules. We suppose a liquid binary mixture A-B having $c_{A}(\equiv c)$ mole of A and $c_{B}$ $\{\equiv(1-c)\}$ mole of $\mathrm{B}$ respectively, where $c_{A}$ and $c_{B}$ are the mole fractions of $\mathrm{A}$ ( $\equiv$ $\mathrm{In})$ and $\mathrm{B}(\equiv \mathrm{Tl})$ in the binary liquid alloy $\mathrm{A}-\mathrm{B}$.

The expression for free energy of mixing $\left(G_{M}\right)$ can be derived on the basis of regular soultion model. It is given as [14]

$$
G_{M}=R T[c \ln c+(1-c) \ln (1-c)]+c(1-c) \cdot \omega
$$

where $R$ is the universal gas constant, $T$ the temperature, and $\omega$ the interaction energy parameter to be determined.

The term $c(1-c) \cdot \omega$ represents the heat of mixing in the frame work of regular solution model, i.e.

$$
H_{M}=\omega c_{A} c_{B}
$$

The activity $a_{A}$ of the element $\mathrm{A}$ in the binary alloys A-B is given by the standard relation

$$
R T \ln a_{A}=G_{M}+(1-c) \frac{\partial G_{M}}{\partial c}
$$

Using Equations (1) and (3) we get the activity of element A as 


$$
\ln a_{A}=\ln c+\frac{\omega}{R T}(1-c)^{2}
$$

Similarly, the activity of the element $\mathrm{B}$ is given by

$$
\ln a_{B}=\ln (1-c)+\frac{\omega}{R T} c^{2}
$$

The temperature derivative of $G_{M}$ provides an expression for integral entropy of mixing $\left(S_{M}\right)$ which is given by

$$
S_{M}=-\frac{\partial G_{M}}{\partial T}
$$

From Equations (1) and (6), we get

$$
\frac{S_{M}}{R}=-[c \ln c+(1-c) \ln (1-c)]-c(1-c) \cdot \frac{1}{R} \frac{\partial \omega}{\partial T}
$$

The relation between free energy of mixing $\left(G_{M}\right)$, entropy of mixing $\left(S_{M}\right)$, and heat of mixing $\left(H_{M}\right)$ is given by a standard thermodynamic relation,

$$
\frac{H_{M}}{R T}=\frac{S_{M}}{R}+\frac{G_{M}}{R T}
$$

Using Equations (1), (7) and (8), we get

$$
\frac{H_{M}}{R T}=c(1-c) \cdot \frac{\omega}{R T}+c(1-c) \frac{1}{R} \cdot \frac{\partial \omega}{\partial T}
$$

\subsection{Structural Properties}

The concentration structure factor or concentration fluctuation in the long wavelength limit $\left(S_{c c}(0)\right)$ which has been used to study the nature of atomic order in binary liquid alloy [17] can also be deduce using regular solution model and is given by using relation,

$$
S_{c c}(0)=\frac{R T}{\left(\frac{\partial^{2} G_{M}}{\partial c^{2}}\right)_{T, P, N}}
$$

From Equations (1) and (10), we get

$$
s_{c c}(0)=\frac{c_{A} c_{B}}{1-2 c_{A} c_{B} \cdot \frac{\omega}{R T}}
$$

The observed value of concentration fluctuation in the long wavelength limit $S_{c c}(0)$ is obtained from observed data of the activities of the constituent species of the binary liquid alloys from the relation

$$
s_{c c}(0)=(1-c) a_{A}\left(\frac{\partial a_{A}}{\partial c}\right)_{T, P, N}^{-1}=c a_{B}\left(\frac{\partial a_{B}}{\partial c}\right)_{T, P, N}^{-1}
$$

where, $a_{A}$ and $a_{B}$ are the activities of the component of $\mathrm{A}$ and $\mathrm{B}$ respectively.

The degree of local order in the liquid mixture is quantified by the Warren-Cowley [18] [19] short range order parameter $\left(\alpha_{1}\right)$. It provides insight into the local arrangement of the atoms in the molten alloys. The theoretical values of 
$\left(\alpha_{1}\right)$ can be evaluated as

$$
\alpha_{1}=\frac{s-1}{s(Z-1)+1}
$$

where, $s=\frac{S_{c c}(0)}{S_{c c}^{i d}(0)}, s_{c c}^{i d}(0)=c_{A} c_{B}$ and $Z$ is the coordination number, which is taken $10(=Z)$ for our purpose.

\subsection{Surface Property}

Butler derived an expression for the surface tension of liquid binary mixture on the basis of the assumption that there exists a mono atomic layer at the surface of a liquid solution, called surface monolayer, as a separate phase that is in thermodynamic equilibrium with the bulk phase [15] [20]. The expression for surface tension, $\Gamma$ on the basis of Butler equation for binary A-B solution at temperature $T$ is given by

$$
\begin{aligned}
\Gamma & =\Gamma_{1}+\frac{1}{A_{1}}\left(G_{1}^{E, s}-G_{1}^{E, b}\right)+\frac{R T}{A_{1}}\left[\ln \left(1-c_{2}^{s}\right)-\ln \left(1-c_{2}^{b}\right)\right] \\
& =\Gamma_{2}+\frac{1}{A_{2}}\left(G_{2}^{E, s}-G_{2}^{E, b}\right)+\frac{R T}{A_{2}}\left[\ln \left(c_{2}^{s}\right)-\ln \left(c_{2}^{b}\right)\right]
\end{aligned}
$$

where, $R$ is universal gas constant. $c_{i}^{s}$ and $c_{i}^{b}$ are mole fraction of component " $i$ " in the surface and bulk respectively. $\Gamma_{1}$ and $\Gamma_{2}$ are the surface tension of the pure component 1 and 2 respectively. $G_{i}^{E, s}$ and $G_{i}^{E, b}(i=1.2)$ are partial excess free energy of component " $i$ " in the surface and the bulk respectively. The molar surface area of the component " $i$ " can be computed by using the relation

$$
A_{i}=K \cdot N_{A}^{1 / 3} \cdot V_{i}^{2 / 3}
$$

where, $K(=1.091)$ is geometrical factor for the liquid alloy, $N_{A}$ is Avogadro's number, $V_{i}\left(=V_{I n}, V_{T l}\right)$ is the molar volume of the component $i$. where, $V_{I n}=V_{M, I n}\left[1+\alpha_{P, I n}\left(T-T_{M, I n}\right)\right]$ and $V_{T l}=V_{M, T l}+\left[1+\alpha_{P, T l}\left(T-T_{M, T l}\right)\right]$

$$
\begin{aligned}
& V_{M, I n}=\text { atomic volume of } \mathrm{In} \text { at melting point } \\
& V_{M, T l}=\text { atomic volume of } \mathrm{Tl} \text { at melting point } \\
& T_{M, I n}=\text { melting temperature of } \mathrm{In} \\
& T_{M, T l}=\text { melting temperature of } \mathrm{Tl} \\
& \alpha_{P, I n}=\text { volume coefficient of In at constant temperature } \\
& \alpha_{P, T l}=\text { volume coefficient of } \mathrm{Tl} \text { at constant temperature }
\end{aligned}
$$

\subsection{Transport Properties}

The diffusion coefficients also provide the insight into the mixing behavior of an alloy in microscopic level. The relation between diffusion coefficient and concentration fluctuation is given as [21]

$$
\frac{D_{M}}{D_{i d}}=\frac{S_{C C}^{i d}(0)}{S_{C C}(0)}
$$


With

$$
D_{M}=c_{1} D_{2}+c_{2} D_{1}
$$

where, $D_{M}$ is the mutual diffusion coefficient and $D_{i d}$ is the intrinsic diffusion coefficient for an ideal mixture; $D_{1}$ and $D_{2}$ are the self-diffusivities of pure components $\mathrm{A}$ and $\mathrm{B}$ respectively.

In term of energy order parameter $\omega$, the diffusion coefficient can be expressed as [22]

$$
\frac{D_{M}}{D_{i d}}=\left[1-\frac{2 \omega}{R T} S_{c c}^{i d}(0)\right] \text { (17) If } D_{M} / D_{i d}>1 \text {, then it indicates compound for- }
$$
mation and if $D_{M} / D_{i d}<1$ then there is tendency of phase separation. For ideal mixing, $D_{M} / D_{i d}$ approaches 1 .

The mixing behavior of liquid alloys at microscopic level can also be understood in terms of viscosity. We have employed the Moelwyn-Hughes equation [16] in order to observe the atomic transport behavior of In-Tl alloy. The Moelwyn-Hughes equation for viscosity of liquid mixture is given as

$$
\eta=\left(c_{1} \eta_{1}+c_{2} \eta_{2}\right)\left(1-c_{1} c_{2} \cdot \frac{H_{M}}{R T}\right)
$$

where, $\eta_{K}$ is the viscosity of pure component $K$ and for most liquid metals, it can be calculated from Arrhenius type equation [21] at temperature $T$ as

$$
\eta_{K}=\eta_{O K} \exp \left[\frac{E_{n}}{R T}\right]
$$

where, $\eta_{O K}$ is constant (in unit of viscosity) and $E_{n}$ is the energy of activation of viscous flow for pure metal (in unit of energy per mole).

\subsection{Optimization of Free Energy of Mixing $\left(G_{M}\right)$, Activity $(a)$, Heat of Mixing $\left(H_{M}\right)$, Concentration Fluctuations $\left(S_{c c}(0)\right)$, Surface Tension and Viscosity}

The model parameters which can be used to optimize thermodynamic, structural and transport properties of the liquid mixture can be obtained by thermodynamic description based on statistical thermodynamics or polynomial expressions. The adjustable coefficients, used in the process, are estimated by least square method which gives an idea to extrapolate into temperature and concentration region in which the direct observed determination is unavailable.

Redlich-Kister polynomial equation gives the composition dependence of excess free energy of mixing and is given by

$$
G_{M}^{X S}(c, T)=c(1-c) \sum_{l=0}^{m} K_{l}(T)[c-(1-c)]^{l}
$$

with

$$
K_{l}(T)=A_{1}+B_{1} T+C_{1} T \ln T+D_{1} T^{2}+\cdots
$$

The coefficients $K_{l}$ are the function of the temperature.

The values for the free energy of mixing $\left(G_{M}\right)$ of In-Tl liquid alloy at different temperatures can be calculated from Equation (1) by knowing the values of ordering energy parameter $(\omega)$ at different temperatures from the relation [23] 


$$
\omega\left(T_{K}\right)=\omega(T)+\frac{\mathrm{d} \omega}{\mathrm{d} T}\left(T_{K}-T\right)
$$

where, $\omega(T)$ is the order energy parameter at the temperature $723 \mathrm{~K}, \omega\left(T_{K}\right)$ is order energy parameter at required temperature $T_{K}$, and $\frac{\mathrm{d} \omega}{\mathrm{d} T}$ represents temperature derivative of order energy parameter which has already been estimated from observed data of entropy of mixing $\left(S_{M}\right)$ of In-Tl liquid alloys at $723 \mathrm{~K}$ from Hultgren et al. 1973 [24].

\section{Results and Discussion}

\subsection{Theoretical Investigation of Different Properties of In-TI Liquid Alloy at $723 \mathrm{~K}$}

The value of main input parameters i.e. interchange energy $(\omega)$ and $\frac{1}{R} \frac{\partial \omega}{\partial T}$ used for the calculation of thermodynamic properties of In-Tl liquid alloy at 723 K were computed from Equations (1) and (9) by using observed values [24] of $G_{M}$ and $H_{M}$ respectively. The best fit parameters were found to be

$$
\frac{\omega}{R T}=0.52, \frac{1}{R} \frac{\partial \omega}{\partial T}=0.15
$$

The positive value of interaction energy indicates that $\mathrm{In}-\mathrm{Tl}$ is segregating in nature. Using above interchange energy $(\omega)$, we computed free energy of mixing $\left(G_{M}\right)$, activity (a) entropy of mixing $\left(S_{M}\right)$, heat of mixing $\left(H_{M}\right)$, concentration fluctuation $\left(S_{c c}(0)\right)$, ratio of diffusion coefficient $\left(D_{M} / D_{i d}\right)$, short-range order parameter $\left(\alpha_{1}\right)$, surface tension $\left(\Gamma_{1}\right)$ and viscosity $(\eta)$ at $723 \mathrm{~K}$ using regular solution model.

The plot of observed and computed values of free energy of mixing with respect to the concentration of $\mathrm{Tl}$ is shown in Figure 1 . The computed and observed values [24] of free energy of mixing are in good agreement in the entire concentration range. Both the computed and observed value of free energy of mixing are minimum at $C_{T l}=0.5$, which indicates that $\mathrm{In}-\mathrm{Tl}$ alloys in liquid state at $723 \mathrm{~K}$ is symmetric at equiatomic composition.

We computed the activities of In and $\mathrm{Tl}$ in the liquid $\mathrm{In}-\mathrm{Tl}$ alloys using the same energy parameter $(\omega)$ as used in Equation (1), and then compared them with observed values as depicted in Figure 2. The plot shows that there is a good agreement between the sets of computed and observed results. Activity coefficient represents the measure of tendency of a component to leave the solution and it provides correlation of the behavior of the systems.

The heat of mixing and entropy of mixing of In-Tl binary liquid alloy at $723 \mathrm{~K}$ were computed from Equations (9) and (7) respectively. To determine heat of mixing $\left(H_{M}\right)$ and entropy of mixing $\left(S_{M}\right)$ we need temperature derivatives of energy parameters. The observed values of heat of mixing [24] were utilized to obtain the temperature derivatives by the successive approximation. The best fit parameters was found to be $\frac{1}{R} \frac{\partial \omega}{\partial T}=0.15$. Both computed and observed values 
of heat of mixing and entropy of mixing are shown in Figure 3 and Figure 4. Both the computed and observed value of heat of mixing and entropy of mixing are maximum at $C_{T l}=0.5$. The positive value of heat of mixing indicates the segregating nature of the alloy which is in agreement with the sign of the interaction energy $\omega$. The value of entropy of mixing is positive in whole range of concentration as depicted in Figure 4.

We computed concentration fluctuation in the long wavelength limit $S_{c c}(0)$ using Equation (11) with the help of same energy parameter $\omega$ for the consistency as used for the computation of free energy of mixing, activity and heat of mixing. The observed $S_{c c}(0)$ were computed from Equation (12). It was found that the computed and observed values of $S_{c c}(0)>S_{c c}^{\text {id }}(0)$ at all compositions. This indicates that segregation is favored in the In- $\mathrm{Tl}$ alloy at $723 \mathrm{~K}$. The computed values of $S_{c c}(0)$ are in good agreement with the observed values in entire concentration range. The theoretical value is maximum at $C_{T l}=0.5$ (i.e. $\left.S_{c c}(0)=0.3378\right)$ as depicted in Figure 5 .

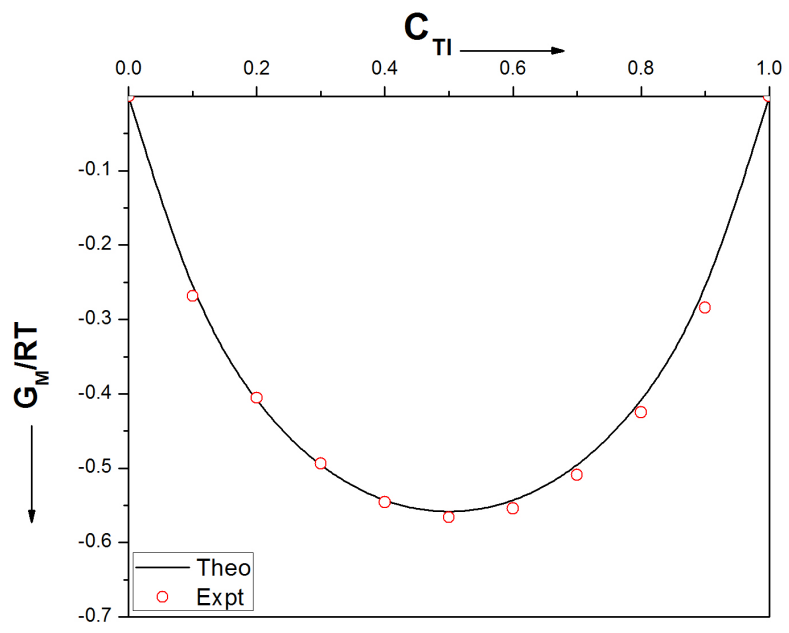

Figure 1. Compositional dependence of $\left(G_{m} / R T\right)_{\exp }$ and $\left(G_{m} / R T\right)_{\mathrm{Th}}$ of In-Tl liquid alloy at $723 \mathrm{~K}$.

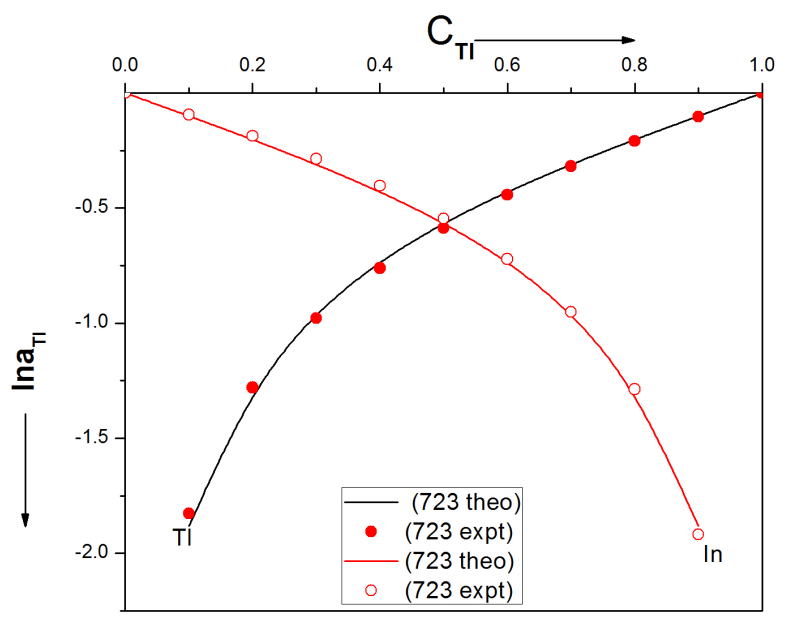

Figure 2. Compositional dependence of $\ln a_{(i) \exp }$ and $\ln a_{(i) \mathrm{Th}}$ of In-Tl liquid alloy at $723 \mathrm{~K}$. 


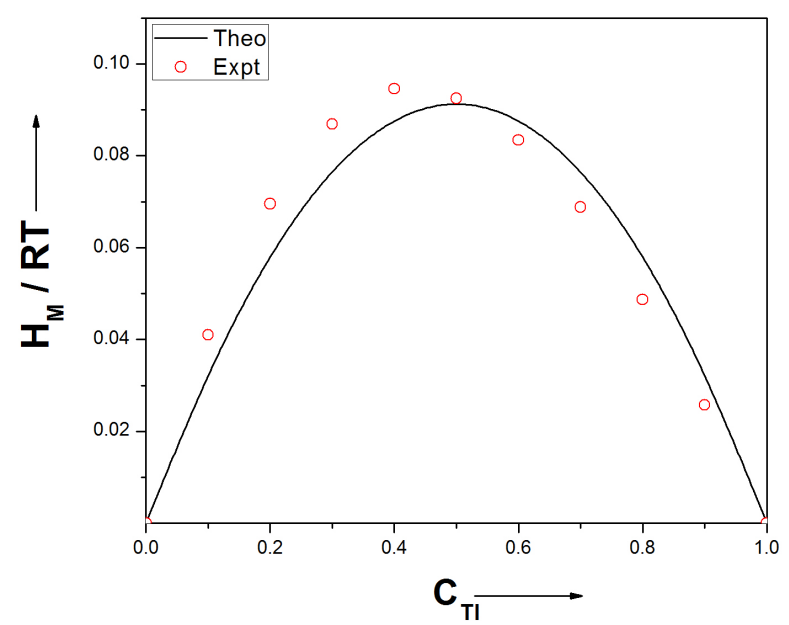

Figure 3. Compositional dependence of $\left(H_{m} / R T\right)_{\exp }$ and $\left(H_{m} / R T\right)_{\mathrm{Th}}$ of In-Tl liquid alloy at $723 \mathrm{~K}$.

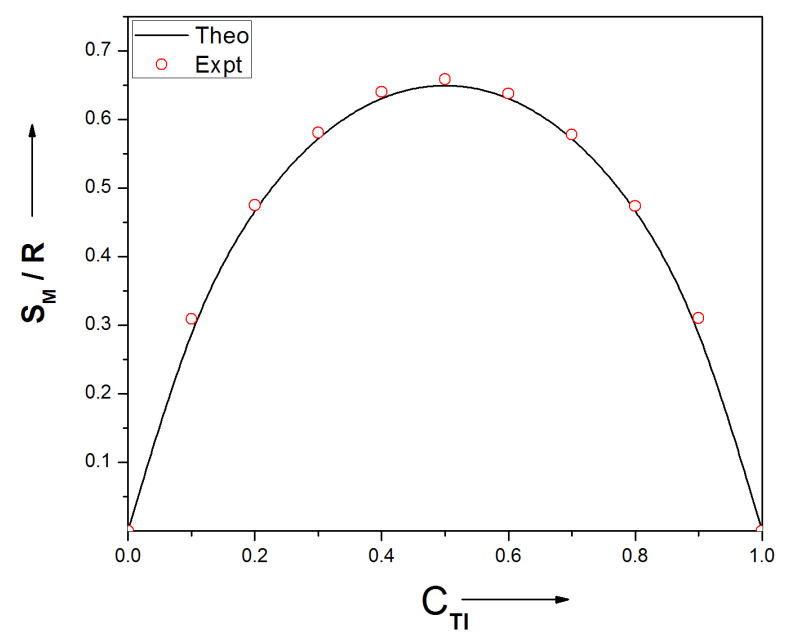

Figure 4. Compositional dependence of $\left(S_{m} / R\right)_{\exp }$ and $\left(S_{m} / R\right)$ of In-Tl liquid alloy at $723 \mathrm{~K}$.

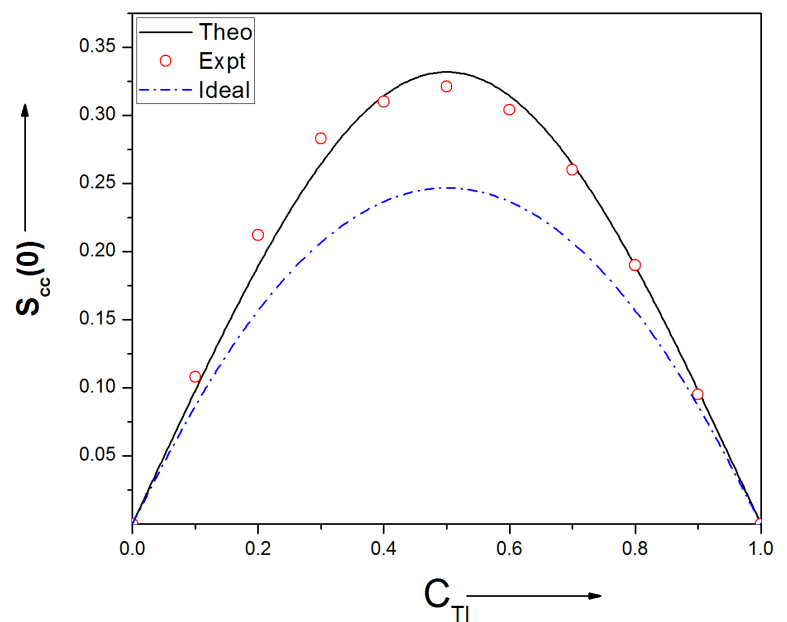

Figure 5. Compositional dependence of $\left(S_{c c}(0)\right)_{\exp }$ and $\left(S_{c c}(0)\right)_{\mathrm{Th}}$ of In-Tl liquid alloy at $723 \mathrm{~K}$. 


\subsection{Theoretical Investigation of In-Tl at $623 \mathrm{~K}, 723 \mathrm{~K}, 800 \mathrm{~K}$ and 923 K by Optimization Method}

By using the best fit value of $\frac{\mathrm{d} \omega}{\mathrm{d} T}$ and $\omega(T)$ at the given temperature $T=723 \mathrm{~K}$ in Equation (22), the value of $\omega\left(T_{K}\right)$ at temperature $T_{K}=623 \mathrm{~K}, 800 \mathrm{~K}, 923 \mathrm{~K}$ are estimated and listed in Table 1.

The values of free energy of mixing $\left(G_{M}\right)$ of In-Tl liquid alloy at different temperatures (i.e. 623,723,800 and $923 \mathrm{~K}$ ) computed by using the corresponding values of $\omega(T)$ in Equation (22) over the entire range of concentration and then they are used to calculate the corresponding excess free energy of mixing $\left(G_{M}^{X S}\right)$ of the alloy at temperature of study by using Equation (20) conjugation with Equations (21) and (22).

The least-square method was used to calculate the parameters involved in Equation (21) and then the optimized coefficients for the alloy are computed which are listed in the Table 2.

We used the parameters i.e. $k_{0}, k_{1}, k_{2}$ and $k_{3}$ to obtain partial excess free energy. The partial excess free energy of mixing $\left(G_{M}^{-X S}\right)$ of the components A (=In) and $\mathrm{B}(=\mathrm{Tl})$ were computed and tabulated below using equations [22]

$$
G_{M, A}^{-X S}(C, T)=(1-C)^{2} \sum_{l=0}^{m} K_{l}(T)[(1+2 l) c-(1-c)](2 c-1)^{l-1}
$$

and

$$
G_{M, B}^{-X S}(C, T)=C^{2} \sum_{l=0}^{m} K_{l}(T)[c-(1+2 l)(1-c)](2 c-1)^{l-1}
$$

The partial excess free energy of mixing of both the components In and $\mathrm{Tl}$ involved in In-Tl liquid alloy at different temperatures (i.e. $=623 \mathrm{~K}, 723 \mathrm{~K}, 800$ $\mathrm{K}, 923 \mathrm{~K}$ ) were calculated separately over the entire concentration range by Equations (23) and (24) with the help of optimized coefficients. With the help of this optimized partial excess free energy of mixing of both the components in the alloy have been used to calculate the corresponding excess free energy of the alloy at different temperatures over the entire range of concentration from the relation

$$
G_{M}^{X S}=C G_{M, A}^{-X S}+(1-C) G_{M, B}^{-X S}
$$

The optimized values of excess free energy of mixing for the alloys at different temperatures (i.e. $=623 \mathrm{~K}, 723 \mathrm{~K}, 800 \mathrm{~K}, 923 \mathrm{~K}$ ) over the entire concentration range are shown in Figure 6.

Now, the activity coefficients $\left(\Upsilon_{i}\right),(i=\mathrm{In}$ or $\mathrm{Tl})$ at different temperature over the entire range of concentration for In or $\mathrm{Tl}$ components have been computed from the relation $\bar{G}_{M, i}^{X S}=R T \ln \Upsilon_{i}$, with $\Upsilon_{i}=\frac{a_{i}}{c_{i}}$, where, $a_{i}$ and $c_{i}$ be the activity and concentration of the component respectively of In-Tl liquid alloy at corresponding temperature.

The calculated optimized values partial excess free energy of mixing, the corresponding activity coefficients and corresponding activity of both the compo- 
nents involved in In-Tl liquid alloy in the entire concentration range at the temperature $T=623 \mathrm{~K}, 723 \mathrm{~K}, 800 \mathrm{~K}, 923 \mathrm{~K}$ are shown in Table 3, Table 4, Table 5 and Table 6.

The concentration fluctuations in long wavelength limit $S_{c c}(0)$ of the alloy at different temperatures in entire concentration range have been calculated using Equation (14) with the help of the optimized values of the activity of both the components which is shown in Figure 7. The values of concentration fluctuation decreases as the temperature of the alloy increases correspond to the concentration of $\mathrm{Tl}$ component. Also, $S_{c c}(0)>S_{c c}^{i d}(0)$ at entire concentration range of $\mathrm{Tl}$ at all temperature of investigation which indicates segregating nature of the alloy.

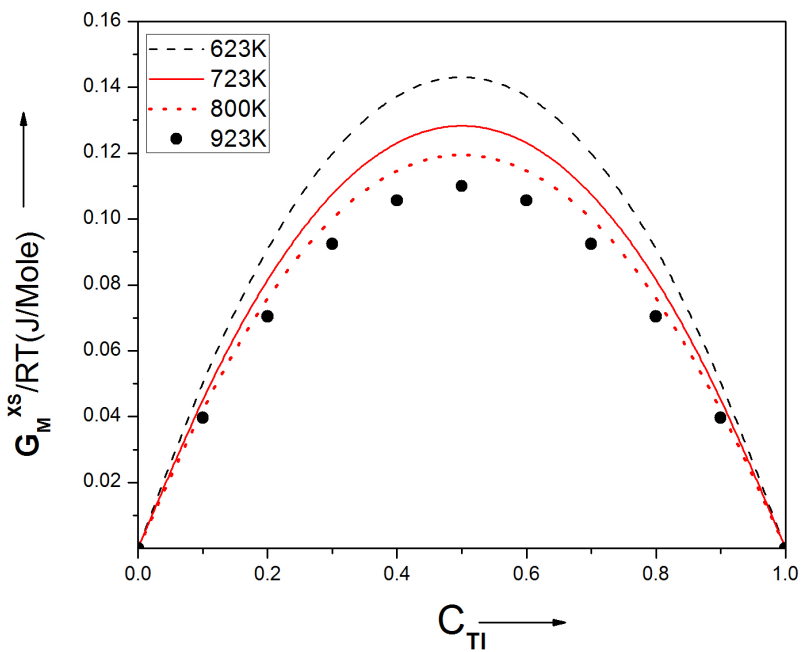

Figure 6. Compositional dependence of $\frac{G_{M}^{X S}}{R T}$ of In-Tl liquid at different temperatures.

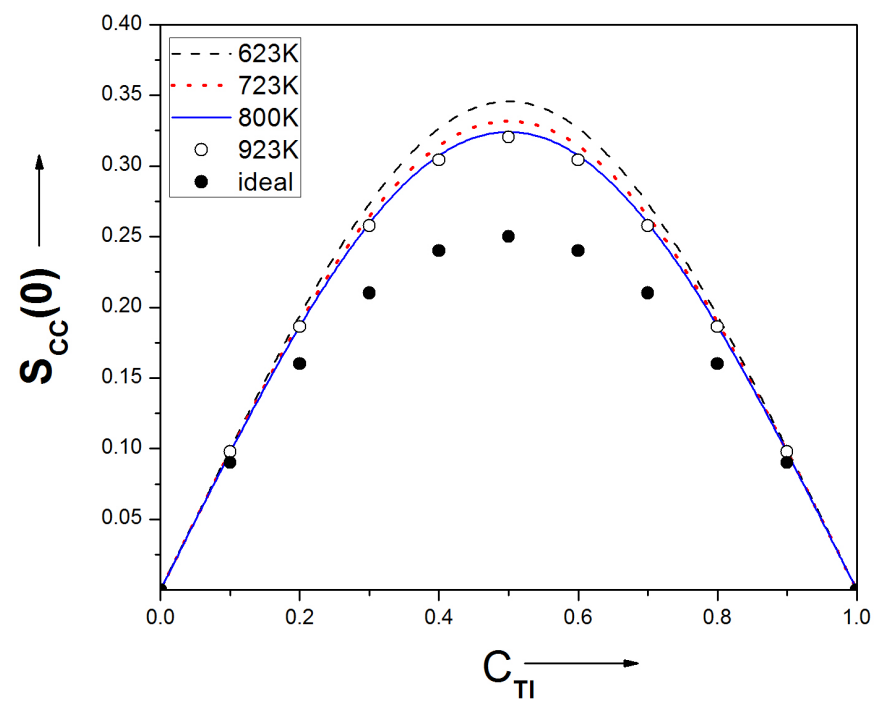

Figure 7. Graph for $S_{c c}(0)$ at different temperatures versus $C_{\mathrm{T} 1}$ of In-Tl liquid alloy (the ideal values in the graph are taken at $723 \mathrm{~K}$ ). 
Table 1. Estimated values of order energy parameter at different temperatures in In-Tl liquid alloy.

\begin{tabular}{cc}
\hline Temperature $\left(T_{K}\right)$ in $\mathrm{K}$ & Order energy Parameter $\omega\left(\mathrm{T}_{\mathrm{K}}\right) / \mathrm{RT}$ \\
\hline 623 & 0.58 \\
723 & 0.52 \\
800 & 0.4843 \\
923 & 0.44 \\
\hline
\end{tabular}

Table 2. Calculated values of optimized coefficients $A_{p}, B_{p} C_{l}$ and $D_{I}(I=0$ to 3$)$ in liquid alloy In-Tl.

\begin{tabular}{ccccc}
\hline Values of 1 & $A_{I}\left(\mathrm{Jmole}^{-1} \mathrm{~K}^{-1}\right)$ & $B_{I}\left(\mathrm{Jmole}^{-1} \mathrm{~K}^{-1}\right)$ & $C_{I}\left(\mathrm{Jmole}^{-1} \mathrm{~K}^{-1}\right)$ & $D_{I}\left(\mathrm{Jmole}^{-1} \mathrm{~K}^{-1}\right)$ \\
\hline 0 & 2.345121281 & -0.020964158 & 0.002942825 & $-1.29189 \mathrm{E}-06$ \\
1 & $3.88327 \mathrm{E}-14$ & $-6.62413 \mathrm{E}-16$ & $9.9353 \mathrm{E}-17$ & $-6.28848 \mathrm{E}-20$ \\
2 & 0 & 0 & 0 & 0 \\
3 & $3.74724 \mathrm{E}-14$ & $-6.89401 \mathrm{E}-16$ & $1.04971 \mathrm{E}-16$ & $-7.35625 \mathrm{E}-20$ \\
\hline
\end{tabular}

Table 3. Optimized values of partial excess free energy of mixing, activity coefficients and activity of both the components involved in In-Tl liquid alloys at $723 \mathrm{~K}$.

\begin{tabular}{cccccccccccc}
\hline \multicolumn{9}{c}{ Tl-Component } & \multicolumn{3}{c}{ In-Component } \\
\hline$C_{\mathrm{Tl}}$ & $\frac{\bar{G}_{M, T l}^{X S}}{R T}$ & $\ln \Upsilon_{\mathrm{Tl}}$ & $\Upsilon_{\mathrm{T} 1}$ & $a_{\mathrm{T} 1}$ & $\operatorname{Ln}\left(a_{\mathrm{Tl}}\right)$ & $\frac{\bar{G}_{M, \text { In }}^{X S}}{R T}$ & $\ln \Upsilon_{\mathrm{In}}$ & $\Upsilon_{\mathrm{In}}$ & $a_{\mathrm{In}}$ & $\operatorname{Ln}\left(a_{\mathrm{In}}\right)$ \\
\hline 0.1 & 0.4212 & 0.4212 & 1.5238 & 0.15238 & -1.8814 & 0.0052 & 0.0052 & 1.0052 & 0.9047 & -0.10016 \\
0.2 & 0.3328 & 0.3328 & 1.3949 & 0.27897 & -1.2766 & 0.0208 & 0.0208 & 1.021 & 0.8168 & -0.20234 \\
0.3 & 0.2548 & 0.2548 & 1.2902 & 0.38706 & -0.9492 & 0.0468 & 0.0468 & 1.0479 & 0.7335 & -0.30987 \\
0.4 & 0.1872 & 0.1872 & 1.2059 & 0.48235 & -0.7291 & 0.0832 & 0.0832 & 1.0868 & 0.6521 & -0.42763 \\
0.5 & 0.1300 & 0.1300 & 1.1388 & 0.56941 & -0.5631 & 0.1300 & 0.1300 & 1.1388 & 0.5694 & -0.56315 \\
0.6 & 0.0832 & 0.0832 & 1.0868 & 0.65206 & -0.4276 & 0.1872 & 0.1872 & 1.2059 & 0.4823 & -0.72909 \\
0.7 & 0.0468 & 0.0468 & 1.0479 & 0.73354 & -0.3099 & 0.2548 & 0.2548 & 1.2902 & 0.3871 & -0.94917 \\
0.8 & 0.0208 & 0.0208 & 1.021 & 0.81681 & -0.2023 & 0.3328 & 0.3328 & 1.3949 & 0.279 & -1.27664 \\
0.9 & 0.0052 & 0.0052 & 1.0052 & 0.90469 & -0.1002 & 0.4212 & 0.4212 & 1.5238 & 0.1524 & -1.88139 \\
\hline
\end{tabular}

Table 4. Optimized values of partial excess free energy of mixing, activity coefficients and activity of both the components involved in In-Tl liquid alloys at $623 \mathrm{~K}$.

\begin{tabular}{cccccccccccc}
\hline \multicolumn{9}{c}{ Tl-Component } & \multicolumn{7}{c}{ In-Component } \\
\hline$C_{\mathrm{T} 1}$ & $\bar{G}_{M, T 1}^{X S}$ & $\ln \Upsilon_{\mathrm{T} 1}$ & $\Upsilon_{\mathrm{T} 1}$ & $a_{\mathrm{T} 1}$ & $\operatorname{Ln}\left(a_{\mathrm{T} 1}\right)$ & $\frac{\bar{G}_{M, l n}^{X S}}{R T}$ & $\ln \Upsilon_{\mathrm{In}}$ & $\Upsilon_{\mathrm{In}}$ & $a_{\mathrm{In}}$ & $\operatorname{Ln}\left(a_{\mathrm{In}}\right)$ \\
\hline 0.1 & 0.4698 & 0.4698 & 1.5997 & 0.15997 & -1.83279 & 0.0052 & 0.0052 & 1.0052 & 0.9047 & -0.10016 \\
0.2 & 0.3712 & 0.3712 & 1.4495 & 0.28989 & -1.23824 & 0.0208 & 0.0208 & 1.021 & 0.8168 & -0.20234 \\
0.3 & 0.2842 & 0.2842 & 1.3287 & 0.39861 & -0.91977 & 0.0468 & 0.0468 & 1.0479 & 0.7335 & -0.30987 \\
0.4 & 0.2088 & 0.2088 & 1.2322 & 0.49288 & -0.70749 & 0.0832 & 0.0832 & 1.0868 & 0.6521 & -0.42763 \\
0.5 & 0.1450 & 0.1450 & 1.156 & 0.57802 & -0.54815 & 0.1300 & 0.1300 & 1.1388 & 0.5694 & -0.56315 \\
0.6 & 0.0928 & 0.0928 & 1.0972 & 0.65835 & -0.41803 & 0.1872 & 0.1872 & 1.2059 & 0.4823 & -0.72909 \\
\hline
\end{tabular}




\section{Continued}

\begin{tabular}{rlllllllllll}
\hline 0.7 & 0.0522 & 0.0522 & 1.0536 & 0.73751 & -0.30447 & 0.2548 & 0.2548 & 1.2902 & 0.3871 & -0.94917 \\
0.8 & 0.0232 & 0.0232 & 1.0235 & 0.81878 & -0.19994 & 0.3328 & 0.3328 & 1.3949 & 0.279 & -1.27664 \\
0.9 & 0.0058 & 0.0058 & 1.0058 & 0.90524 & -0.09956 & 0.4212 & 0.4212 & 1.5238 & 0.1524 & -1.88139 \\
\hline
\end{tabular}

Table 5. Optimized values of partial excess free energy of mixing, activity coefficients and activity of both the components involved in In-Tl liquid alloys at $800 \mathrm{~K}$.

\begin{tabular}{cccccccccccc}
\hline \multicolumn{7}{c}{ Tl-Component } & \multicolumn{7}{c}{ In-Component } \\
\hline$C_{\mathrm{Tl} 1}$ & $\bar{G}_{M, T l}^{X S}$ & $\ln \Upsilon_{\mathrm{Tl}}$ & $\Upsilon_{\mathrm{Tl}}$ & $a_{\mathrm{Tl}}$ & $\operatorname{Ln}\left(a_{\mathrm{Tl}}\right)$ & $\frac{\bar{G}_{M, L n}^{X S}}{R T}$ & $\ln \Upsilon_{\mathrm{In}}$ & $\Upsilon_{\mathrm{In}}$ & $a_{\mathrm{In}}$ & $\operatorname{Ln}\left(a_{\mathrm{In}}\right)$ \\
\hline 0.1 & 0.3923 & 0.3923 & 1.4804 & 0.14804 & -1.9103 & 0.0048 & 0.0048 & 1.0049 & 0.9044 & -0.10052 \\
0.2 & 0.3100 & 0.3100 & 1.3634 & 0.27267 & -1.2995 & 0.0194 & 0.0194 & 1.0196 & 0.8156 & -0.20377 \\
0.3 & 0.2373 & 0.2373 & 1.2678 & 0.38035 & -0.9667 & 0.0436 & 0.0436 & 1.0446 & 0.7312 & -0.31309 \\
0.4 & 0.1743 & 0.1743 & 1.1905 & 0.47619 & -0.7419 & 0.0775 & 0.0775 & 1.0806 & 0.6483 & -0.43334 \\
0.5 & 0.1211 & 0.1211 & 1.1287 & 0.56435 & -0.5721 & 0.1211 & 0.1211 & 1.1287 & 0.5644 & -0.57207 \\
0.6 & 0.0775 & 0.0775 & 1.0806 & 0.64834 & -0.4333 & 0.1743 & 0.1743 & 1.1905 & 0.4762 & -0.74194 \\
0.7 & 0.0436 & 0.0436 & 1.0446 & 0.73119 & -0.3131 & 0.2373 & 0.2373 & 1.2678 & 0.3803 & -0.96667 \\
0.8 & 0.0194 & 0.0194 & 1.0196 & 0.81565 & -0.2038 & 0.3100 & 0.3100 & 1.3634 & 0.2727 & -1.29949 \\
0.9 & 0.0048 & 0.0048 & 1.0049 & 0.90437 & -0.1005 & 0.3923 & 0.3923 & 1.4804 & 0.148 & -1.9103 \\
\hline
\end{tabular}

Table 6. Optimized values of partial excess free energy of mixing, activity coefficients and activity of both the components involved in In-Tl liquid alloys at $923 \mathrm{~K}$.

\begin{tabular}{|c|c|c|c|c|c|c|c|c|c|c|}
\hline \multicolumn{6}{|c|}{ Tl-Component } & \multicolumn{5}{|c|}{ In-Component } \\
\hline$C_{\mathrm{Tl}}$ & $\frac{\bar{G}_{M, T l}^{X S}}{R T}$ & $\ln \Upsilon_{\mathrm{Tl}}$ & $\Upsilon_{\mathrm{Tl}}$ & $a_{\mathrm{T} 1}$ & $\operatorname{Ln}\left(a_{\mathrm{T1}}\right)$ & $\frac{\bar{G}_{M, I n}^{X S}}{R T}$ & $\ln \Upsilon_{\text {In }}$ & $\Upsilon_{\text {In }}$ & $a_{\text {In }}$ & $\operatorname{Ln}\left(a_{\text {In }}\right)$ \\
\hline 0.1 & 0.3564 & 0.3564 & 1.3619 & 0.13619 & -1.9937 & 0.0044 & 0.0044 & 1.0044 & 0.904 & -0.10096 \\
\hline 0.2 & 0.2816 & 0.2816 & 1.2764 & 0.25529 & -1.3654 & 0.0176 & 0.0176 & 1.0178 & 0.8142 & -0.20554 \\
\hline 0.3 & 0.2156 & 0.2156 & 1.2055 & 0.36164 & -1.0171 & 0.0396 & 0.0396 & 1.0404 & 0.7283 & -0.31707 \\
\hline 0.4 & 0.1584 & 0.1584 & 1.1472 & 0.45886 & -0.779 & 0.0704 & 0.0704 & 1.0729 & 0.6438 & -0.44043 \\
\hline 0.5 & 0.1100 & 0.1100 & 1.1100 & 0.55002 & -0.5978 & 0.1100 & 0.1100 & 1.1163 & 0.5581 & -0.58315 \\
\hline 0.6 & 0.0704 & 0.0704 & 1.0629 & 0.63775 & -0.4498 & 0.1584 & 0.1584 & 1.1716 & 0.4687 & -0.75789 \\
\hline 0.8 & 0.0176 & 0.0176 & 1.0154 & 0.8123 & -0.2079 & 0.2816 & 0.2816 & 1.3252 & 0.265 & -1.32784 \\
\hline 0.9 & 0.0044 & 0.0044 & 1.0038 & 0.90344 & -0.1015 & 0.3564 & 0.3564 & 1.4282 & 0.1428 & -1.94619 \\
\hline
\end{tabular}

The natural logarithms of optimized values of activity of both the components in the alloy at different temperatures in the entire concentration range are shown in Figure 8.

The Warren-Cowley chemical short-range order parameter $\alpha_{1}$ is used to explain the degree of local arrangement of atoms in the mixture. The chemical 
short-range order parameter was computed from Equation (13) using coordination number, $Z=10$ for liquid the alloy at $723 \mathrm{~K}$. The value of short range order parameter has been found positive in all concentration range which indicates that the alloy is segregating at all compositions. The value of short range order parameter has been found maximum at $C_{\mathrm{Tl}}=0.5$ at $723 \mathrm{~K}$ as shown in Figure 9. We have computed chemical short-range order parameter at temperatures $723 \mathrm{~K}$, $623 \mathrm{~K}, 800 \mathrm{~K}$ and $923 \mathrm{~K}$. The value of short range order parameter has been found maximum at $C_{\mathrm{Tl}}=0.5$ at all temperature of study as shown in Figure 9. The plot depicts that $\alpha_{1}$ is positive in the entire range of concentration showing that $\alpha_{1}$ in In-Tl is segregating.

The surface tension of the liquid alloys can be computed using Equation (14) conjugation with Equations (15) and (16) using Buttler's model [15] [20] [25]. The ratio of partial excess Gibbs energy in the bulk and that in the surface can be expressed as $\beta=\frac{G_{i}^{E, s}}{G_{i}^{E, b}}, \beta$ has been taken as 0.83 [26] [27]. We have taken the surface tension of In-Tl and temperature coefficients for pure In and $\mathrm{Tl}$ components from the reference [28]. The surface tension of the pure component at temperature of investigation has been calculated using the relation.

$\Gamma(T)=\Gamma_{m}+\frac{\partial \Gamma}{\partial T}\left(T-T_{m}\right)$, where, $\frac{\partial \Gamma}{\partial T}\left(=-0.09 \mathrm{mNm}^{-1} \mathrm{~K}^{-1}\right.$ for In, $-0.08 \mathrm{mN}$ $\mathrm{m}^{-1} \mathrm{~K}^{-1}$ for $\left.\mathrm{Tl}\right)$ is temperature coefficient of surface tension, $\mathrm{Tm}(=430 \mathrm{~K}$ for In and $577 \mathrm{~K}$ for $\mathrm{Tl}$ ) is melting temperature and $T=723 \mathrm{~K}$.

The partial excess free energy of mixing of the pure components was taken from Hultgren et al. [24]. It was found from the analysis that the computed surface tension for In-Tl system at $723 \mathrm{~K}$ is less than ideal value $\left(=X_{1} \Gamma_{1}+X_{2} \Gamma_{2}\right)$ at all the concentration of In i.e. there is negative departure of surface tension from ideality at $723 \mathrm{~K}$. It was found that surface tension increases with increase in the concentration of component $\mathrm{Tl}$ on the alloy as shown in the Figure 10.

To find the surface tension $(\Gamma)$ of the alloy at different temperature we have used the values of optimized partial excess free energy i.e. $\bar{G}_{M}^{X S}$ from the Tables 3-6, and using Buttler's Equation (16) conjugation with Equation (17). The calculated values of surface tension at different temperatures are shown in Figure 10. The surface tension of the alloy in the concentration range of $\mathrm{Tl}$ component showed that as the temperature increases, the surface tension decreases.

Viscosity of pure component $\mathrm{In}$ and $\mathrm{Tl}$ at $723 \mathrm{~K}$ are calculated using Equation (19) which is required to compute viscosity of In-Tl alloy at $723 \mathrm{~K}$ with the help of the value of the constants $E$ and $\eta_{o k}$ for the metals [28]. To compute viscosity using Moelwyn-Hughes equation [16], heat of mixing $H_{M}$ is required which is taken from regular solution model calculations. The result of viscosity at $723 \mathrm{~K}$ of the alloy with the ideal values $\left(\eta=c_{A} \eta_{A}+c_{B} \eta_{B}\right)$ is shown in Figure 11. The graph shows that with the increase in concentration of $\mathrm{Tl}$ component in the alloy, the viscosity of the alloy decreases (Figure 11).

The optimized viscosity of the alloys is calculated by the Moelwyn-Hughes 
Equation (22) conjugation with Equation (23). The values of optimized $\mathrm{H}_{\mathrm{M}} / \mathrm{RT}$ is used in whole concentration range obtained using Equation (11) with the help of optimized values of $\omega(T)$ and $\frac{\mathrm{d} \omega}{\mathrm{d} T}$ at temperature of study. The values of parameters on Equation (23) is taken from [28]. The plot exhibits that the viscosity show small negative deviation from ideality at all compositions at $723 \mathrm{~K}$. The computed values of viscosity at different temperatures is as shown in Figure 11.

The diffusion coefficients $\frac{D_{M}}{D_{i d}}$ of In-Tl liquid alloy at temperatures of study in the entire concentration range can also be calculated using the optimized data from the relation (19) which are shown in the Figure 12. The $D_{M} / D_{i d}<1$ which indicates that there is tendency of phase separation.

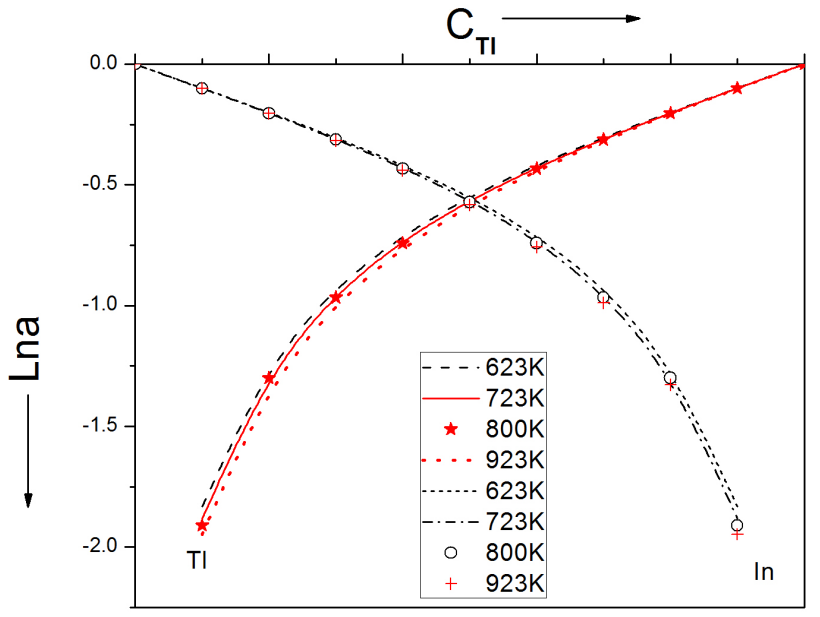

Figure 8. Graph for $\ln a$ at different temperatures versus $C_{\mathrm{Tl}}$ of In-Tl liquid.

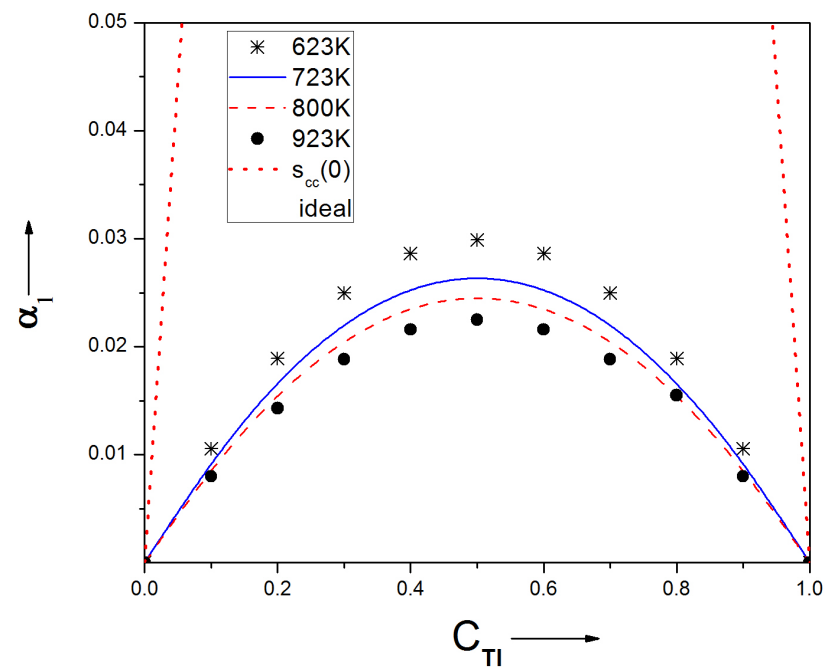

Figure 9. Graph for short range order parameter i.e. $\alpha_{1}$ at different temperatures versus $C_{\mathrm{T} 1}$ of In-Tl liquid alloy (the ideal values in the graph are taken at $723 \mathrm{~K}$ ). 


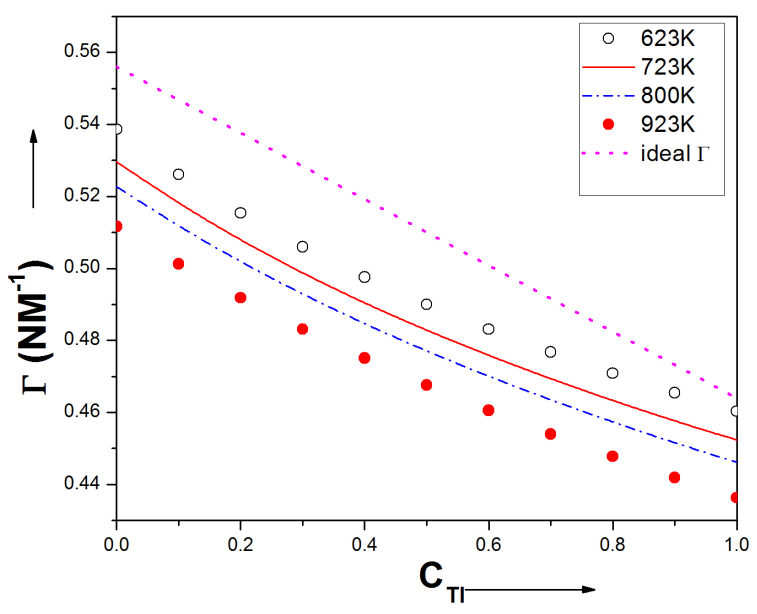

Figure 10. Graph for surface tension i.e. $\Gamma$ at different temperatures versus $C_{\mathrm{Tl}}$ of In-Tl liquid alloy (the ideal values in the graph are taken at $723 \mathrm{~K}$ ).

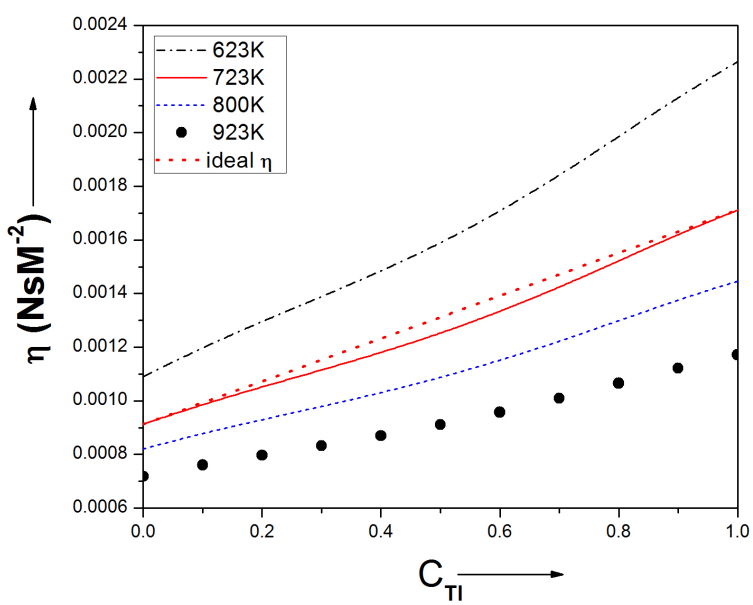

Figure 11. Graph for viscosity i.e. $\eta$ at different temperatures versus $C_{\mathrm{Tl}}$ of In-Tl liquid alloy (the ideal values in the graph are taken at $723 \mathrm{~K}$ ).

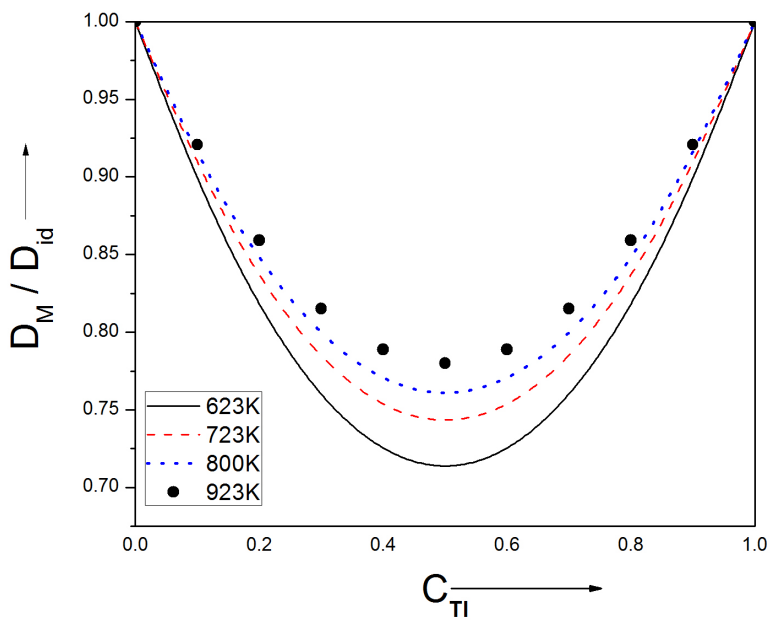

Figure 12. Graph for diffusion coefficient i.e. $\frac{D_{M}}{D_{i d}}$ at different temperatures versus $C_{\mathrm{Tl}}$ of In-Tl liquid alloy. 


\section{Conclusion}

From the theoretical investigation following conclusions can be drawn:

1) Symmetry is observed in free energy of mixing, heat of mixing and entropy of mixing at all temperatures of investigations.

2) Activity decrease with the increase in concentration of $\mathrm{Tl}$ component in all temperatures of study i.e. $623 \mathrm{~K}, 723 \mathrm{~K}, 800 \mathrm{~K}$ and $923 \mathrm{~K}$. And, with compare to temperature of study, it decreases as temperatures of investigation increases.

3) At all temperatures of investigation, the surface tension decrease with the increase of bulk concentration of $\mathrm{Tl}$ in Tl-In liquid alloy. In context of different temperatures, as temperature of study increases, it decreases.

4) Viscosity increases as the concentration of $\mathrm{Tl}$ component increases in the alloy. And, as temperature of study increases viscosity also increases.

5) The diffusion coefficients i.e. $D_{M} / D_{i d}<1$ at all compositions which indicates that there is tendency of phase separation

\section{Acknowledgements}

Authors are grateful to University Grants Commission, Nepal for providing financial support to pursue this work under Faculty Research Grant Program-2017.

\section{Conflicts of Interest}

The authors declare no conflicts of interest regarding the publication of this paper.

\section{References}

[1] Jordan, A.S. (1970) A Theory of Regular Associated Solution Applied to the Liquid us Curves of the Zn-Te and Cd-Te System. Metallurgical Transactions, 1, 239-249.

[2] Bhatia, A.B. and Hargrove, W.H. (1974) Concentration Fluctuation and Thermodynamic Properties of Some Compound Forming Binary Molten Alloys. Physical Review B, 10, 3186-3196. https://doi.org/10.1103/PhysRevB.10.3186

[3] Bhatia, A.B. and Singh, R.N. (1982) Short Range Order and Concentration Fluctuations in Regular and Compound Forming Molten Alloys. Physics and Chemistry of Liquids, 11, 285-313. https://doi.org/10.1080/00319108208080752

[4] Sommer, F. (1990) Thermodynamic Properties of Compound-Forming Liquid Alloys. Journal of Non-Crystalline Solids, 117-118, 505-512. https://doi.org/10.1016/0022-3093(90)90580-F

[5] Prasad, L.C., Chatterjee, S.K. and Singh, V.N. (1996) Intermetallic Associations in AlMg Liquid Alloys. Physica B, 217, 285-291. https://doi.org/10.1016/0921-4526(95)00885-3

[6] Novakovic, R., Muolo, M.L. and Passerone, A. (2014) Bulk and Surface Properties of Liquid X-Zr (X=Ag, Cu) Compound Forming Alloys. Surface Science, 549, 281-293. https://doi.org/10.1016/j.susc.2003.12.006

[7] Adhikari, D., Jha, I.S. and Singh, B.P. (2002) Mixing Properties of Al-Mg Liquid 
Alloys. Indian Journal Physics, 86, 783-786. https://doi.org/10.1007/s12648-012-0113-3

[8] Jha, N. and Mishra, A.K. (2001) Thermodynamic and Surface Properties of Liquid Mg-Zn Alloys. Journal of Alloys and Compounds, 329, 224-229.

https://doi.org/10.1016/S0925-8388(01)01684-X

[9] Koirala, R.P., Kumar, J., Singh, B.P. and Adhikari, D. (2014) Bulk and Surface Properties of Co-Fe and Fe-Pd Liquid Alloys. Journal of Non-Crystalline Solids, 394-395, 9-15. https://doi.org/10.1016/j.jnoncrysol.2014.04.001

[10] Luo, Z.P. (2012) An Overview on the Indium-Thallium (In-Tl) Shape Memory Alloy Nanowires, Metallography. Microstructure, and Analysis, 1, 320-326. https://doi.org/10.1007/s13632-012-0046-4

[11] Koirala, I., Singh, B.P. and Jha, I.S. (2014) Theoretical Assessment on Segregating Nature of Liquid In-Tl Alloys. Journal of Non-Crystalline Solids, 398-399, 26-31. https://doi.org/10.1016/j.jnoncrysol.2014.04.018

[12] Shrestha, G.K., Singh, B.K. and Jha, I.S. (2018) Theoretical Investigations on Temperature Dependence of Thermodynamic Properties and Concentration Fluctuations of In-Tl Binary Liquid Alloys by Optimization Method. BIBECHANA, 5, 11-23.

[13] Wayman, C.M. and Shimizu, K. (1972) The Shape Memory ("Marmem") Effect in Alloys. Metal Science Journal, 6, 175-183.

https://doi.org/10.1179/030634572790446028

[14] Guggenheim, E.A. (1952) Mixture. Oxford University Press, Oxford.

[15] Butler, J.A.V. (1932) The Thermodynamics of the Surfaces of the Solutions. Proceedings of the Royal Society A: Mathematical, Physical \& Engineering Sciences, 135, 348-375. https://doi.org/10.1098/rspa.1932.0040

[16] Moelwyn-Hughes, E.A. (1974) Physical Chemistry. Longmans Green and Co., Oxford, London.

[17] Bhatia, A.B. and Singh, R.N. (1982) Thermodynamic Properties of Compound Forming Molten Alloys in a Weak Interaction Approximation. Physics and Chemistry of Liquids, 11, 343-351. https://doi.org/10.1080/00319108208080755

[18] Warren, B.E. (1969) X-Ray Diffraction. Addison-Wesley, Reading.

[19] Cowley, J.M. (1950) An Approximate Theory of Order in Alloys. Physical Review, 77, 669-675. https://doi.org/10.1103/PhysRev.77.669

[20] Novakovic, R. (2010) Thermodynamics, Surface Properties and Microscopic Functions of Liquid Al-Nb and Nb-Ti Alloys. Journal of Non-Crystalline Solids, 356, 1593-1598. https://doi.org/10.1016/j.jnoncrysol.2010.05.055

[21] Singh, R.N. and Sommer, F. (1998) Thermodynamic Investigation of Viscosity and Diffusion in Binary Liquid Alloys. Physics and Chemistry of Liquids, 36, 17-28. https://doi.org/10.1080/00319109808035917

[22] Singh, R.N. and Sommer, F. (1997) Segregation and Immiscibility in Liquid Binary Alloys. Reports on Progress in Physics, 60, 57-150. https://doi.org/10.1088/0034-4885/60/1/003

[23] Singh, R.N. and Bhatia, A.B. (1984) Flory's Formula for the Entropy of Mixing of NACS Alloy. Journal of Physics F: Metal Physics, 14, 2309-2314. https://doi.org/10.1088/0305-4608/14/10/009

[24] Hultgren, R., Desai, P.D., Hawkens, D.T., Gleisser, M. and Kelly, K.K. (1973) Selected Values of the Thermodynamic Properties of Binary Alloys. ASM, Metal, 430. 
[25] Kaptay, G. (2005) A Unified Equation for the Viscosity of Pure Liquid Metals. Zeitschrift für Metallkunde, 96, 1-8. https://doi.org/10.3139/146.018080

[26] Yeum, K.S., Speiser, R. and Poirier, D.R. (1989) Estimation of the Surface Tensions of Binary Liquid Alloys. Metallurgical Transactions B, 20, 693-703. https://doi.org/10.1007/BF02655927

[27] Tanaka, T., Hack, K., Lida, I. and Hara, S. (1996) Evaluation of Binary Phase Diagrams of Small Particle Systems. Zeitschrift für Metallkunde, 87, 380-389.

[28] Brandes, E.A. and Brook, G.B. (1992) Smithells Metals Reference Book. 7th Edition, Butterworth-Heinemann Linacre House, Jordan Hill, Oxford. 